\title{
Ophthalmic Plexus
}

National Cancer Institute

\section{Source}

National Cancer Institute. Ophthalmic Plexus. NCI Thesaurus. Code C53057.

A small, autonomic nerve plexus located in the head that arises from the internal carotid plexus and penetrates the orbit along the ophthalmic artery. 